\title{
Uma Ferramenta Livre e ExtensíVel Para Detecção de Vulnerabilidades em Sistemas Web
}

\author{
Douglas Rocha ${ }^{1, *}$, Diego Kre utz ${ }^{1}$, Rogério Turche tti ${ }^{2}$ \\ ${ }^{1}$ Grupo de Pesquisa em Sistemas de Informação, GPSI Universidade Federal do Pampa, UNIPAMPA Alegrete, RS, Brasil \\ ${ }^{2}$ Colégio Técnico Industrial de Santa Maria, CTISM Universidade Federal de Santa Maria, UFSM Santa Maria, RS, Brasil
}

\begin{abstract}
The increasing number of intrusions and data thefts on online systems is one of the triggers of the growing concern about security inside organizations. Nowadays, dynamic and extensible detection tools are required and critical to detect and diagnose vulnerabilities in Web systems. In this paper we present the development and evaluation of a vulnerability scanner for online systems. Unlike most existing tools, it is free and open source, available at SourceForge, and has a modular and extensible arch itecture. The achieved results show that the proposed tool, called Un iscan, is able to better detect and diagnose vulnerabilities such as LFI, RFI and RCE.
\end{abstract}

Keywords Vu lnerability Detection, Security, Web Systems, Free, Open Source Tools, Flexible, Extensible Architecture

\section{Introdução}

Sistemas Web (online) são alvos fáceis e frequentes para os atacantes,principalmente por oferecerem serviços ligados diretamente à Internet[1, 2, 3, 4,5].A lgumas pesquisas indicam que o número de vulnerabilidades e ataques é cada vez maior e mais frequente em serviços e sistemas $\operatorname{Web}[3,4$, 5,6,7]. Mesmo aqueles sistemas que oferecem serviços onlinemenos atraentes são alvo de ataques, pois os servidores que hospedam os sistemas online vulneráveis servem para os atacantes como pontes para consequentesataques às vítimas mais visadas, como aquelas que possuem dados e informações financeiras de clientes. Logo, é visível a crescente necessidade de boas práticas de segurança no contexto de sistemas $\mathrm{Web}[8,9]$. Isso inclui, com especial atenção, a detecção e o diagnóstico de vulnerabilidades nos sistemas. Este é o primeiro passo para um bom ciclo de desenvolvimento, evolução e manutenção dos sistemas online. A detecção deve ainda considerar, nos processos de testes de segurança, casos em que o comportamento da aplicação seja imprevisível aos dados de entrada[10]. Esta característica torna os testes de segurança uma tare fa não trivial.

Testar manualmente todas as possíveis vulnerabilidades de sistemas Web é u ma tarefa complexa e mu ito dispendiosa, sendo considerada como impraticável. Devido a esse fato pode-se observar, tanto no contexto científico quanto

* Corresponding author:

douglas.poerschke@gmail.com (Douglas Rocha)

Published online at http://journal.sapub.org/computer

Copyright (C) 2012 Scientific \& Academic Publishing. All Rights Reserved pro-fissional, a eminente necessidade de ferramentas de análise de segurança que executem testes automáticos, como os diferentes scanners de vulnerabilidades $[1,3,5,11,12]$. Boa parte dessas soluções são baseadas em banco de dados de vulnerabilidades conhecidas e utilizam rastreadores, também denominados decrawlers, para encontrar os links e subsistemas do sitio $\mathrm{Web}$ a ser analisado e diagnosticado.

A maioria das ferramentas existentes não é capaz de encontrar todas as vulnerabilidades dos sistemas online[11]. Os próprios resultados da varredura para um mesmo sistema alvo, na forma de dados estatísticos e relatórios, variam entre os diferentesscanners de vulnerabilidades[11, 12, 13]. Algumas dessas ferramentas detectam ma is vulnerabilidades que outras, tanto em termos quantitativos para uma mes ma vulnerabilidade, com múltiplas e diversificadas incidências num mesmo sistema, quanto em relação a variabilidade de defeitos detectáveis. Adicionalmente, estudos recentes demonstram que a média de detecção de vulnerabilidades das principais soluções existentes é relativa mente baixa [11].

Boa parte dos scanners de vulnerabilidades atua na detecção devulnerabilidadescomo SQL Injection e Cross-site scripting (XSS) [2, 3, 14].Não obstante, a maior parte das ferramentase xistentessãocomercia is, levando a uma carência de ferramentaslivres/gratuitas eque sejam capazes de contribuir efetiva e eficazmente na tarefa de diagnosticar e melhorar a segurança dos sistemas Web [3,11].

Estudosmostramqueexistemdiferentestipos de ataques contra sistemas Web. Entre osma is frequentesestãoa inclusão remota de arquivos, SQL Injection e cross-scripting[15]. Não obstante, existem outros tipos de vulnerabilidades, comoformasavançadas e de segundaordem de XSS e SQL-i, variações de Cross-Channel Scripting eCross-Site Request Forgery, que aindaestãodescobertaspelasferramentas existen 
tes [11]. Osresultadosde diferentes pesquisas [8, 9, 11, 12, 13] apontamqueaindaháespaçoparainvestigação, inovação e desenvolvimentona área de scanners de vulnerabilidades voltados paraaplicações Web.

Este artigoapresenta o desenvolvimento e os resultados de umscanner de vulnerabilidades livre e gratuito, denominado Uniscan[16], para ser utilizado por profissionais responsáveis pelo desenvolvimento ou segurança de sistemas Web. O objetivo da ferramenta éautomatizar o processo de detecçãoe diagnósticode vulnerabilidades nesses ambientes. Comoprincipais pontos positivos do scannerpodem ser destacados:

(a) u ma ferramenta livre e gratuitamente disponível;

(b) uma arquitetura simples, modular e extensível, per-mitindo a rápida e dinâmica incorporação de novas funcio-nalidades;

(c) incorporação de plug-ins para detectar vulnerabilidades como RFI (Remote File Inclusion), LFI (Local File Inclusion), RCE (Remote Command Execution), SQL-i, XSS e Blind SQL-i;

(d) arquitetura multi-thread para acelerar o processo de análise e diagnóstico detalhado dos sitios Web alvos;

(e) configurabilidade, permitindo ao utilizador selecionar os plug-insde análise desejados para cada sistema alvo;

(f) módulo de stress para testar a resistência das aplicações Web em diferentes níveis, como a configuração dos servidores Web responsáveis por manter os sistemasdisponíveis; e

(g) utilização de metodologia híbrida, através da combina-ção de testes dinâmicos e estáticos.

Vulnerabilidades como RFI, LFI e RCE são pouco exploradas e analisadas pela maioria das soluções existentes. Sendo assim, detectar eficazmente essas três faltas, que podem colocar sistemas $\mathrm{Web}$ em risco, foi um dos objectivos primários do desenvolvimento do Uniscan. Consequente-mente, o scanner pode ser considerado uma iniciativa que contribui com o desenvolvimento de detectores de vulnerabi-lidades capazes de eficientemente detectar vulnerabilidades como LFI, RFI e RCE.

A maioria dos scanners existentes, diferentemente da ferramenta desenvolvida, não possuem uma arquitetura modular e nem suporte a uma metodologia híbrida, utilizando tanto testes estáticos quanto dinâmicos na varredura dos sistemas alvo.A arquitetura modular, flexívele extensível do Un iscanextendea sua utilização e extensão para diferentescenários e casos de aplicação.

Os resultados atingidos demonstram como a ferramenta desenvolvida contribui com o processo de detecção de vulne-rabilidades em sistemas reais. O Uniscan está disponível para downloadem http://sourceforge.net/projects/uniscan/.A fer-ramenta nasceu e evoluiu como resultado de um trabalho acadêmico de pesquisa e desenvolvimento. A versão 5.3 é constituída por cinco grandes módulos e vinte plug-ins.Até o mo mento da escrita deste artigo, foram registrados mais de 6.000 downloadsda ferramenta, tendo como origem mais de 100 paises[17].
O restante do artigo está organizado como segue. A próxima seção apresenta uma breve descrição e caracterização de vulnerabilidades e metodologias de detecção. Na sequência, seção III,sãodiscriminadas a arquitetura e a implementação da ferramenta.Os experimentos e resultados analíticos e estatísticos são apresentados e discutidos na seção IV. Por fim, as inferências finais co mpõe a última seção.

\section{Vulnerabilidades e Metodologias de Detecção}

Esta seção introduz as vulnerabilidades e metodologias de detecção. A primeira sub-seção apresenta exemplos concretos de vulnerabilidades em códigos PHP e Perl, que são alvos iniciais de investigação deste trabalho. A segunda sub-seção aborda as metodologias estática e dinâmica de varredura dos sitios alvo.

\subsection{Vulner abilidades em Códigos Web PHPe Perl}

RFI, LFI e RCE são algumas das vulnerabilidades mais comuns que programadores tendem a inconscientemente implementar sem ter ciência dos riscos envolvidos tanto para as aplicações Web quanto para os dados e sistemas opera-cionais hospedeiros. Elas estão entre as vulnerabilidades presentes com relativa frequência em sistemas Web[15] e representam um nível de risco crítico[24].Conforme estatísticas do Zone-H[25], que registra ataques a servidores e sites na Internet, a maior parte dos servidores Web que foram invadidos em 2010 foi por meio de uma dessas três vulnera-bilidades. Isso evidencia e justifica a importância de detectar, diagnosticar e tratar, da melhor forma possível, tais vulnerabi-lidades.

A vulnerabilidade RFI ocorre quando a validação dos dados, em algu mas funções e procedimentos dos códigos dos sistemas Web, inexiste ou está incompleta. Um exemplo de validação é o processo de verificar se o arquivo que está sendo incluído no código realmente está no diretório especificado pela ap licação Web. Arquivos, em códigos PHP, são comu-mentemente passados como parâmetros $\mathrm{e}$ utilizados em fun-çõescomo include(); include_once(); require(); e require_once(). Geralmente, quando alguma destas funções é uti-lizada, o objetivo é incluir um arquivo local, armazenado em algu m diretório especifico, ao código fonte do PHP[24], agregando código externo.

A vulnerabilidade RFI pode ser explorada por u $m$ atacante quando os parâmetros do código PHP não estão sendo devi-damente verificados. Nesses casos, o atacante pode passarum arquivo qualquer como parâmetro. Co mo exemp lo, considere o endereço eletrônico, conhecido como URL,http://www.exemplo.com/index.php? inc=topo.php.O atacante pode tentar manipular os dados que são passados como parâmetros para o arquivo index.php. Neste caso, ao invés de incluir o arquivo topo.phpno parâmetro inc, o atacante poderia incluir um endereço Web de um código malicioso, como inc $=h t t p: / / w w w . s i t e c r a c k e r . c o m / c m d . t x t ? c$ 
$m d=i d$.Logo,quando o inter-pretador PHP analizar o arquivo index.php, o arquivo cmd.txt, hospedado em www.sitecracker.com, será carregado e incluído no processamento do código. O próprio PHP cria e gerencia a conexão com o sitio malicioso para a download do arquivo remoto.

A Figura 1 ilustra o processo de exploração da vulnera-bilidade RFI. O cracker, atacante, pode utilizar tanto um navegador Web bem como exploits para conseguir explorar essa vulnerabilidade nos scripts PHP do site www.example.com, hospedados no servidor Web do respectivo domínio. Para isso ele necessitará de outro site (exemplo: www.sitecracker.com), hospedado em outro servidor Web, para disponibilizar o seu arquivo malicioso (exemplo: cmd.txt), que servirá para explorar a vulnerabilidade RFI. O resultado do processo é a abertura de uma linha de comando (u ma espécie de shell) via navegador Web, onde, a partir da manipulação dos parâmetros, é possível a execução de comandos no sistema operacional do servidor Web a partir do código PHP.

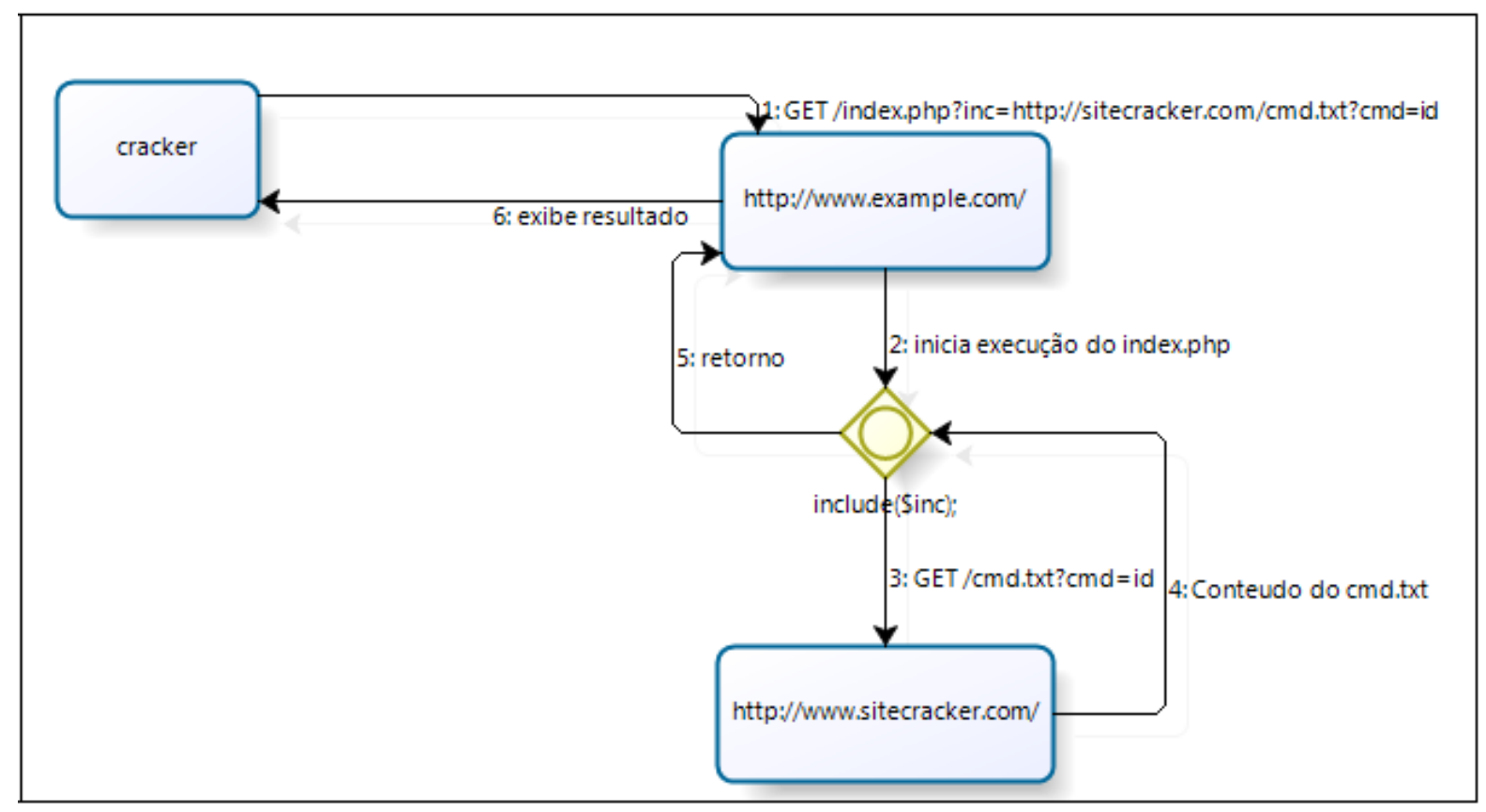

Figura 1. P rocesso de exploração da vulnerabilidade RFI

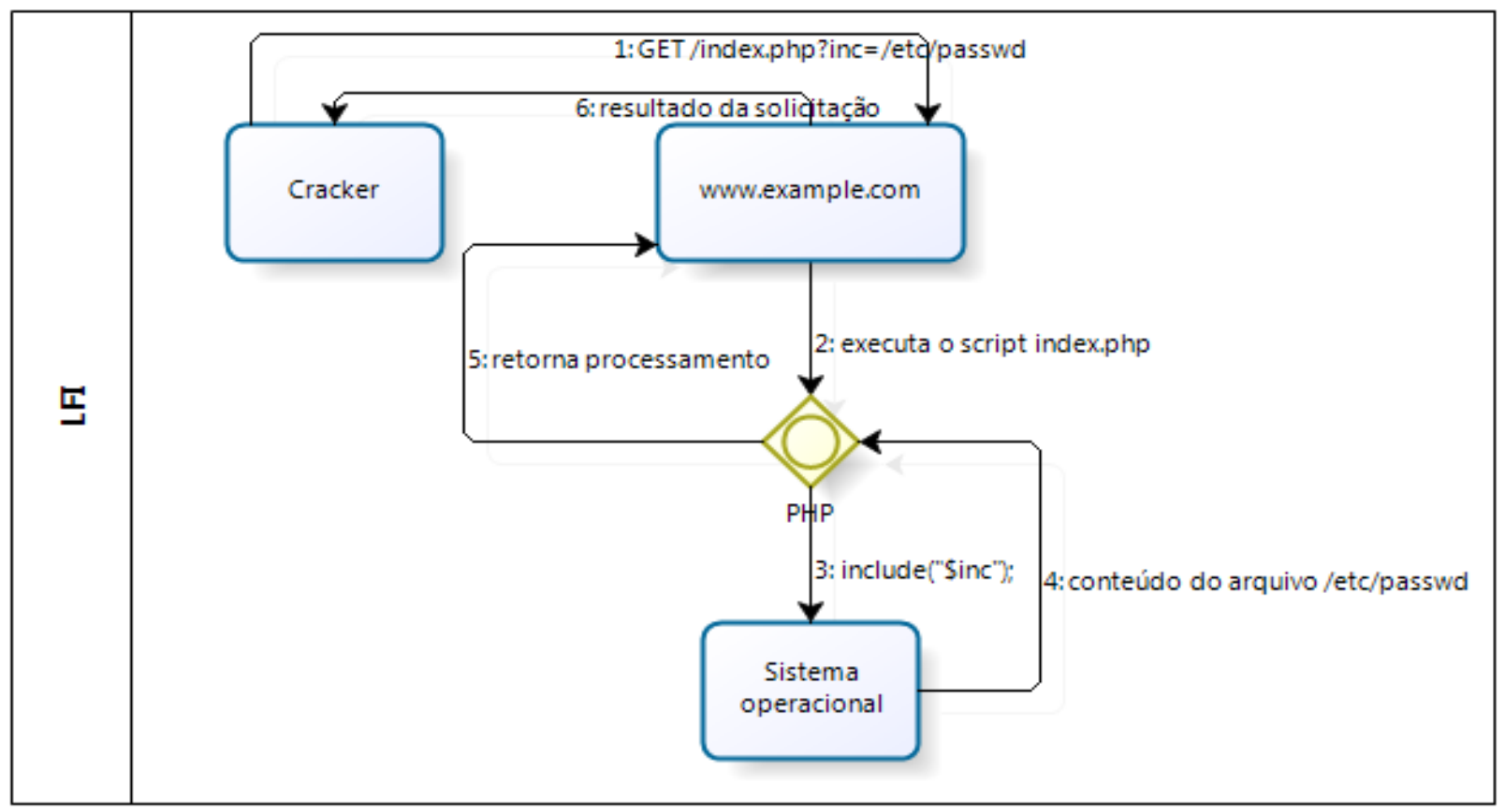

Figura 2. Processo de exploração da vulnerabilidade LFI 
Com frequência, os servidores Web executam em modo usuário, ou seja, não pos suem privilég ios para fazer qualquer coisa no sistema operacional hospedeiro. Mesmo assim, o atacante terá acesso a todas as informações que os níveis de permissão do servidor Web permitem ter acesso. Entretanto, no caso de o servidor Web estar executando em modo super-usuário (root) a situação se torna ainda mais crítica, pois todos os comandos que o atacante utilizar serão executados com permissões de root, tendo acesso completo e irrestrito ao sistema operacional. Em ambos os casos o atacante pode utili-zar o sistema local para atacar outros sistemas internos ou externos aos domínios da entidade sendo atacada.

A vulnerabilidade LFI, assim como a RFI, também é explorada através dos parâmetros de funções como include(), include_once(), require(), e require_once(). Contudo, diferen-temente da RFI, a inclusão é de arquivos locais e não de arqui-vos remotos. O resultado desta vulnerabilidade éo acesso a arquivos locais ao sistema operacional hospedeiro, como o arquivo /etc/passwd, que contém in formações dos usuários do sistema GNU/Linux (index.php?inc=/etc/passw d).

A Figura 2 apresenta o processo de exploração da vulnera-bilidade de LFI. O atacante pode utilizar um navegador Web ou um exploit para explorar essa vulnerabilidade nos scripts PHP do site www.example.com. $\mathrm{O}$ cracker faz uma requisição GET index.php? inc=/etc/passwd para o endereço $w w w$. example.com, o que provoca a inclusão do arquivo passwdna execução do código PHP.

Através do processo ilustrado na Figura 2, um atacante pode exp lorar a vulne rabilidade de inclusão localde arquivos através de um simples navegador, digitando uma URL comohttp://www.example.com/index.php? inc=/etc/passwd. Com es-sa simples ação o conteúdo do arquivo /etc/passwd será incluído no arquivo index.phpe será exibido no navegador Web do atacante. Esses dados podem permitir, por exemp lo, a um atacante lançar um ataque de força bruta para tentar descobrir (decifrar através de tentativa e erro) a senha de cada usuário listado no arquivo /etc/passwd.

Outro exemplo de como explorar a vulnerabilidade LFI é através da utilização dos recursos e arquivos de registro de atividades (logs) do servidor Web. O servidor Web Apache, assim como outros, pode registraros logs dos acessos. Uma forma de utilizar esse recurso é forçar a gravação de logs (comandos) que levarão, posteriormente, a execução de comandos pela inclusão do arquivo de log no cógido PHP. Uma forma de forçar o registro de comandos é pelo simples método de tentar acessar páginas inexistentes com os parâmetros desejados (exemplo: larquivoXYZ.php? id=<? php\%20system("uname $-a$ ”);?>).Isto fará com que a requisição seja gravada no access_log do apache. A partir deste ponto,basta incluir o arquivo access $\log$ na página vulnerável para que o comando uname - a s eja executado no sistema hospe-deiro. A URL para exploração da vulnerabilidade ficaria da seguinte forma: $\quad h t t p: / / w w w . e x a m p l e . o r g /$ index.php? inc $=/$ varl log/apache/access log.Quando incluído no código PHP o arquivo access_log do Apache, este executará o comando uname - $a$ no sistema operacional e mostrará o resultado do comando no navegador do atacante.

$\mathrm{Na}$ execução remota de comandos (RCE) é possível executar chamadas ao sistema hospedeiro através de funções como system e exec. Aslinguagem Perle PHP, em especial, podem apresentar vulnerabilidades quando existiremparâ-metros sem as devidas validações e verificações. As funções system, open e exec da linguagem Perl podem ser classificadas entre as maiores potencializadoras deste tipo de vulnera-bilidade. Em síntese, o problema reside na possibilidade que o código fonte fornece para executar comandos de chamadas ao sistema sem o tratamento adequado dos parâmetros passados à essas funções.

Uma simples linha de código, como a ilustrada a seguir, utilizando a função open() do Perl, pode levar a uma vulne-rabilidade crítica. O uso da função é geralmente destinado à leitura e escrita em arquivos através de operadores como "<", para leitura, e ">", para escrita. No entanto, o uso da função sem os operadores podem levar a outros problemas, co mo a execução de comandos quaisquer.

Exemplo de código Perl vulnerável:

open(\$arq, "Sarquivo”);

No exemplo apresentado, a falta do operador de leitura ("<") ou escrita (">") permite utilizar outros operadores, como pipe (“|"), pois o nome do arquivo será recebido por parâmetro. O operador pipe serve para mudar a entrada padrão da função open(). No caso, a entrada padrão da função open() pode ser alterada para a saída (da execução) padrão de qual-quer outro comando.

A seguir é apresentado um exemplo de exploração desta vulnerabilidade. Ele cons is te em pas sar como parâmetro um pipe,juntamente com um comando a ser executado, ao invés do nome do arquivo.

Exemplo de explo ração do código Perl vulnerável:

http://www.ex.com/script.cgi? arquivo $=\mid$ cat $\% 20 /$ etc/pass $w d \mid$

Com isso, o código Perl, ao executar a função open(), abrirá o pipe e executará o co mando cat / etc/passwd.Isso fará com que o conteúdo do arquivo seja exibido na tela do navegador do atacante. O resultado para a execução do código Perl ficaria:open(\$arq, "|cat /etc/passwd|");.

Na Figura 3 é ilustrado um segundo exemplo, agora PHP, de código vulnerávela RCE. Ao acess ar a página que contém esse código o usuário poderá passar por parâmetro o valor da variável nLinhas. A princípio, isso significa que o funciona-mento do comando tail poderá, pegando mais ou menos linhas do arquivo.txt. Como é realizada uma chamada system, com execução de comandos semelhante a um shell, é possível executar mais de um comando numa única linha. Para is so existe um separador especial “;”, que permite ao usuário introduzir vários comandos na sequência em uma única linha de execução. Isso significa que é possível incluir comandos além do valor da variável nLinhas. Acrescentando ";" seria possível executar uma sequência qualquer de 
comandos devido à falta de verificação do valor da variável nLinhas. A seguiré apresentando umexemplo de inclusão do comando cat.

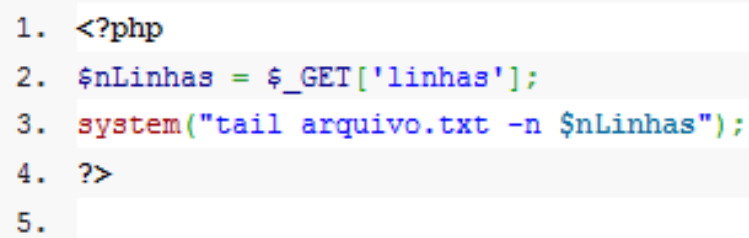

Figura 3. Exemplo de código PHP vulnerável a RCE

Exemplo de alteração do valor da variávelnLinhas: http://www.example.com/index.php? nLinhas $=15$

Exemplo de alteração do valor da variável nLinhascom a inclusão de um segundo comando a ser executado pela função system:

http://www.example.com/index.php? $n$ Linhas $=15$; cat $/$ etc /passwd

O resultado da chamada system ficaria:

system("tail arquivo.txt -n 15 ;cat letc/passwd");

No mo mento em que a função system for invocada, tanto o comando tail quanto o comando cat serão executados sequen-cialmente.No exemplo, o comando cat irá trazer o conteúdo do arquivo /etc/passwd para a tela do navegador do atacante. Uma das formas de resolver o problema de falta de verificação da variável $n$ Linhas é através da inclusão de uma conversão de tipo de dados, também conhecida como casting, com o objetivo de forçar que o valor de nLinhas seja um número inteiro, ou seja, evitando que venha a ser uma cadeia de caracteres (string) com instruções potencialmente maliciosas e prejudicia is ao sistema Web.

As vulnerabilidades LFI, RFI e RCE são exploradas e diagnosticadas de forma similar em códigos PHP e Perl. Estas duas linguagens são amplamente utilizadas na construção de sistemas Web. Esse foi um dos motivos daescolhadessas linguagens de programação como o alvos iniciais para o desenvolvimento dos primeiros plug-ins do Uniscan.

\subsection{Metodologias de Detecção}

Existem basicamente dos tipos de testes utilizados na detecção de vulnerabilidades em sistemas Web, o estático e o dinâmico. A metodologia estática possui algumas vantagens em relação ao método dinâmico, entre elas: (a) ideal para testes de softwares de prateleira (softwares que não são feitos sob encomendas), pois sem uma customização, os nomes de arquivos e variáveis do sistema Web são conhecidos; (b) verifica a existência de arquivos que não estão ligados (exemp lo: mesmo não existindo um link para o arquivo $\mathrm{X}$ no sistema Web, este será testado se estiver no banco de dados do scanner). Co mo desvantagens podem ser apontadas: (i) as diversas requisições de arquivos que não existem no servidor Web; e (ii) a falta de capacidade para analizar páginas de um software customizado, o que é co mu m em sistemas online.

$\mathrm{Na}$ metodologia dinâmica o scannervasculha o sistema e servidor Web alvo a procura de todos os arquivos existentes. Em outras palavras, antes de iniciar os testes de vulnera-bilidades, o próprio scannercriaa lista dos arquivos existentes no servidor Web através de um crawler (rastreador). Todas as verificações de vulnerabilidades são realizadas sobre a lista de arquivos descobertos pelo crawler. A vantagem do método dinâmico é a possibilidade de descobrir erros em arquivos distintos nos sistemas Web, sendo a melhor opção para var-reduras em serviços e sistemas customizados.A desvantagem é o fato de não cons eguir detectar arquivos não ligados e, cons equentemente, não encontrados pelo crawler. No caso do método estático existe uma base de dados com conjuntos de arquivos pré-definidos, os quais serão testados no sistema Web, havendo ou não ligação com o sistema alvo. Portanto, a combinação dos dois métodos pode tirar proveito das vanta-gens de ambos.

\section{Arquitetura do Uniscan}

Um scanner de vulnerabilidades possui módulos essen-ciais, como orastreador e overificador de vulnerabilidades. O rastreador tenta localizar todos os arquivos e links dentro do sitio alvo. Por outro lado, o verificador de vulnerabilidades é responsável por realizar diferentes tipos de testes sobre cada arquivo ou link encontrado. Cada tipo de teste pode ser incorporado à ferramenta como um plug-in, tornando-a fle-xível e extensível.

Outra característica importante incorporada ao Uniscan é o suporte a multip rogra mação ( $m u l t i$-threads), au mentando o desempenho e a capacidade de execução de verificações si-multâneas em sites co mplexos, que podem conter centenas, ou mes mo milhares, de arquivos e links a serem testados.

A Figura 4 ilustra arquitetura projetada e implementadano Uniscan, em sua versão 5.3. Co mo pode ser observado, ela é composta por cinco grandes módulos com funções dife-renciadas. Os módulos contam com o suporte de plug-ins, ou seja, componentes menores e acopláveis ao módulo, trazendo extensibilidade e flexibilidade ao scanner.

\subsection{Descrição dos Módulos}

O primeiro módulo, setup, é responsável pela configuração e inicialização do Uniscan. Ele realiza a leitura dos arquivos de configuração e prepara os parâmetros para os outros módulos, como a definição do número de threads a serem ativadas no sistema.

O módulodiscovery engine gera lis tas de sites, do domínio alvo, utilizando máquinas de busca, como Google e Bing. Estas listas são utilizadas pelo scanner no processo de verificação das vulnerabilidades. Os resultados de buscadores como o Google podem ser interessantes pelo fato de, even-tualmente, conseguirem mapear arquivos ou subsistemas ilhados dentro do sitio alvo, que possam não ser localizadas pelo crawler do scanner devido a quebra ou falta de refe-rências explícitas. 


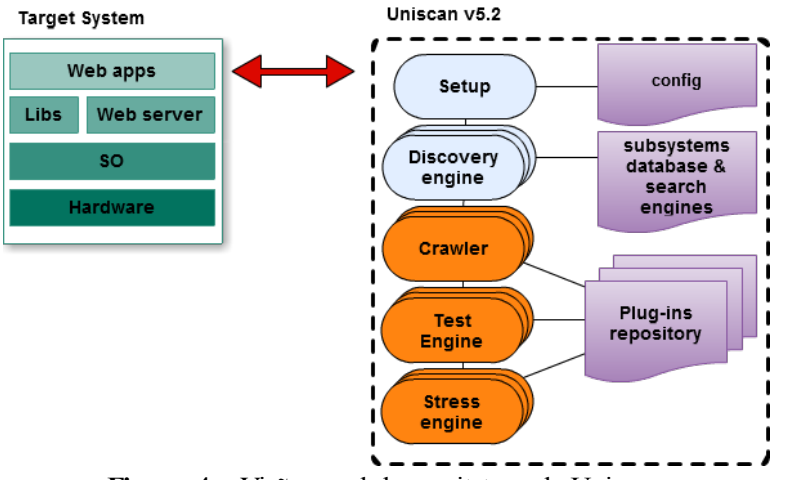

Figura 4. Visão geral da arquitetura do Uniscan

Um dos principais módulosda arquitetura é o crawler (rastreador), sendo responsável por identificar todas as páginas do sistema alvo, através de um processo exaus tivo de varredura. O crawler conta ainda com um repositório de plug-ins para a coleta de informações sensíveis, como email de administradores e comentários no HTML,que possam tornar o sistema vulnerável pela potencialidade de auxiliarem no processo de penetração do atacante. A Figura 5 ilustra o flu xo de operação do crawler.

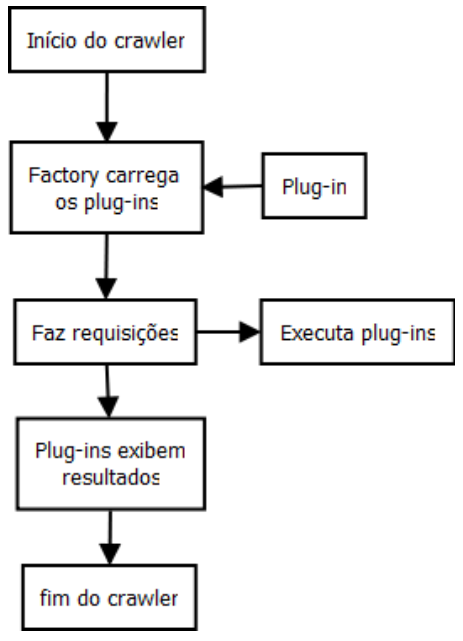

Figura 5. Diagrama do crawler

Omódulo de testes, ou test engine, é responsável pelo carregamento, gerenciamento e execução dos plug-ins de testes de vulnerabilidades. Cada teste é representado por um plug-in armazenado no respositório. A principal tare fa do módulo é carregar e executar todos os plug-ins requisitados pelo usuário. A Figura 6 descreve o funcionamento, na forma de diagrama, do módulo de testes.

O stress engine é responsável por carregar, gerenciar e executar os plug-ins de testes de stress do sistema e do servidor alvo. O objetivo é verificar a estabilidade e disponibilidade de um sistema Web sob variadas cargas de atividade, visando identificar problemas de projeto, imple-mentação ou configuração dos sistemas.

Os recursos e as funcionalidades disponibilizadas pelos módulos da arquitetura do Uniscan são implementados através de plug-ins. Sendo assim, a arquitetura e o repositório de plug-ins trás benefícios como:

(a) a não necessidade de uma nova versão do scanner a cada erro corrigido em um dos plug-ins;

(b) não é necessário lançar u ma nova versão do scanner para adicionar novos testes de vulnerabilidades;

(c) o usuário pode desenvolver seus plug-ins sem a neces-sidade de modificar o núcleo da ferramenta;

(d) maior diversidade e flexibilidade, pois podem ser criadas e testadas diferentes variantes de um teste para uma vulnerabilidade em específico;

(e) maior extensibilidade e longevidade, pois novas fun-cionalidades e testes, inicialmente não previstos, podem ser facilmente agregados à ferramenta; e

(f) maior adaptabilidade, pois a ferramenta pode ser facil-mente personalizada para diferentes cenários e casos de teste.

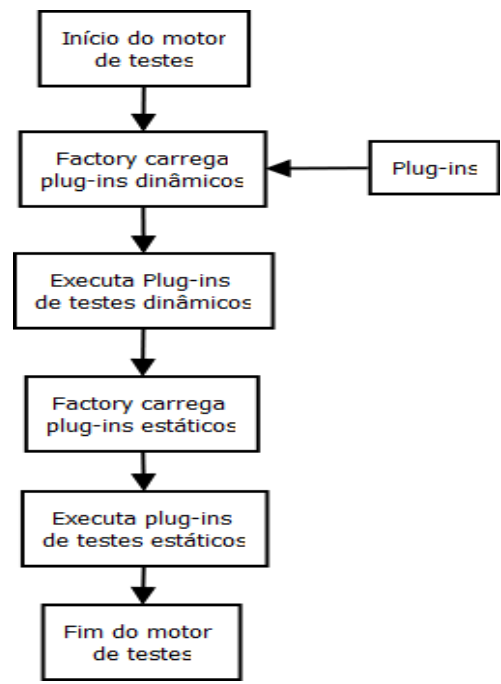

Figura 6. Diagrama do motor detestes

\subsection{Imple mentação}

O Uniscan foi desenvolvido na linguagemde programação Perl, que é uma linguagem presente em vários sistemas ope-racionais. Como consequência direta, a ferramenta é um scanner multiplataforma. Os módulos e plug-ins são estrutu-rados em módulos Perl com a utilização do recurso de factory da linguagem.

O módulo Factory.pm é responsável por carregar cada plug-in do scanner e retornar um objeto deste para a área onde ele foi chamado. Devido a essa função, ele é um dos núcleos importantes da implementação da ferramenta.

O módulo Crawler.pm realiza a navegação e a coleta de dados no sistema alvo. Para cada requisição de entrada, todos os plug-ins habilitados são executados, recebendo como parâ-metros a URL e o seu respectivo conteúdo. Ao final da exe-cução, o método showResults de cada plug-in é invocado para exibir o resultado da correspondente análise.

O módulo Scan.pm é o motor de testes do scanner. A sua entrada é a lista de URLs encontradas pelo crawler.A função do módulo é executar todos os testes (plug-ins) requisitados pelo usuário, inclu indo testes dinâmicos ou estáticos.

A Figura 7 apresenta o diagrama da implementação do Uniscan. Ela ilustra a ordem e as principais co-relações dos módulos e repositórios de plug-ins. 


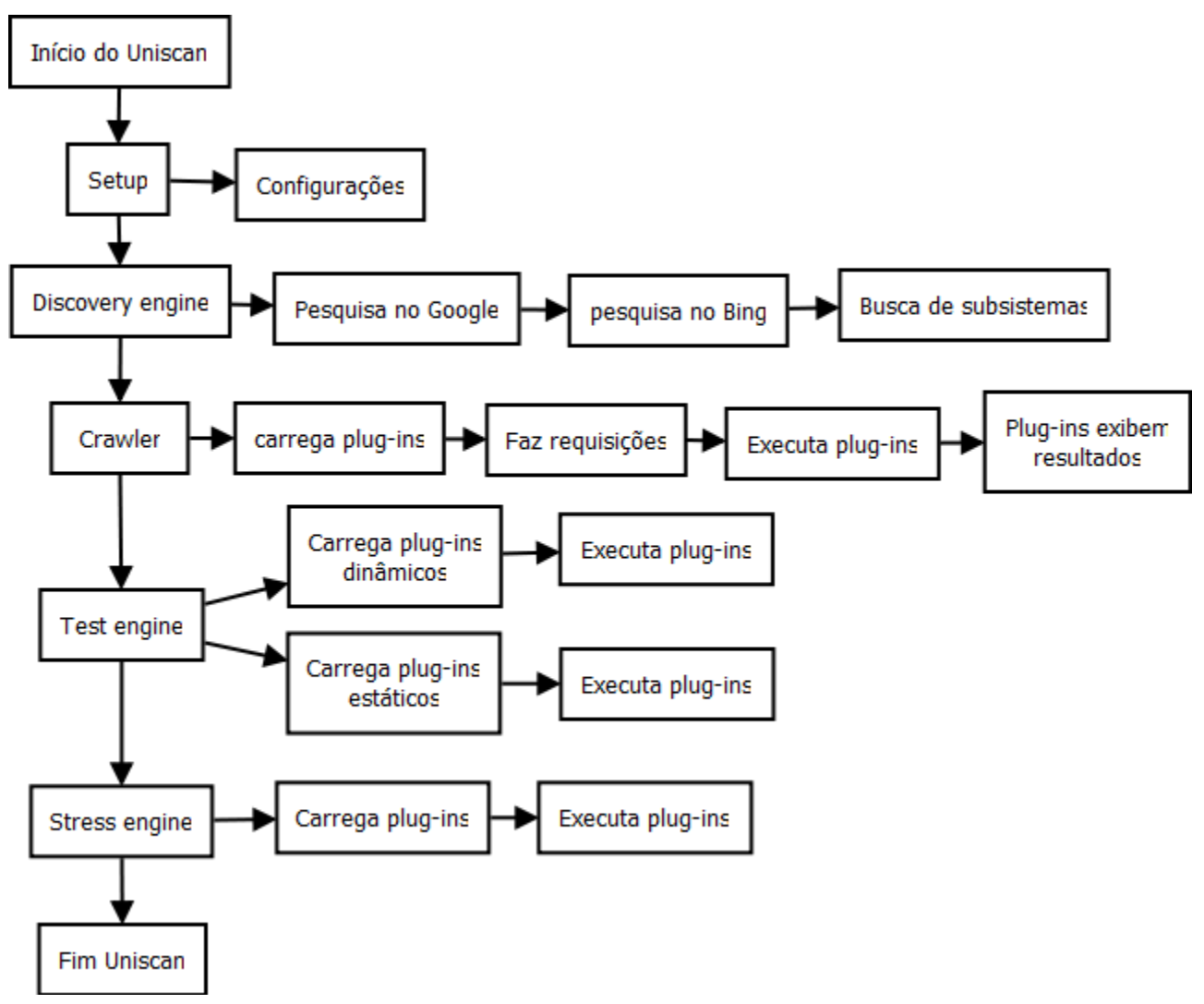

Figura 7. Diagrama geral da implementação do Uniscan

\section{Experimentos Realizados}

Nesta seção são brevemente apresentadas as principais ferramentas similares utilizadas nos testes. Adicionalmente, os cenários de testes são descritos, bem como os resultados obtidos com os experimentos realizados. Foram definidos e utilizados dois tipos de cenários, os controlados e os reais. O primeiro grupo teve como função uma validação e melhor avaliação e comparação inicial das ferramentas. Por outro lado, o segundo grupo visou verificar a efetividade das ferramentas em cenários reais, ou seja, sistemas Web (de empresas e outras organizações) em linha de produção.

\subsection{Ferramentas Similares}

Foram escolhidas e utilizadas seis ferramentas similares para análise de características e funcionalidades, bem como comparação de resultados em cenários de testescontrolados e reais. As ferramentas escolhidas foram: Acunetix[23], Nikto[22], Powerfuzzer[21], Shadow Security Scanner (Shadow)[20], WebCruiser[19] e Websecurity[18].As razões da escolha podem ser definidas como: (a) acesso livre e gratuito a uma cópia do scannerpara análise e avaliação; (b) similaridade de funcionalidades e objetivos; e (c) percepção de utilização no mercado. $O$ primeiro ite $m$ foi u m dos fatores determinanentes na escolha das ferramentas. Adicionalmente, foram utilizadas também outras três ferramentas em dois casos específicos de teste.

No conjunto das ferramentas avaliadas, o Acunetix é consi-derado uma dos melhores scanners de vulnerabilidades do mercado[11]. Com isso, é possível concluir que a escolha das ferramentas é adequada aos objetivos pretendidos, como a comparação de funcionalidades e capacidade de detecção dos tipos de vulnerabilidades alvo do Uniscan.

A Tabela 1 apresenta os resultados da comparação inicial de funções e recursos dos scanners de vulnerabilidades. O " $\mathrm{X}$ " indica a capacidade de detecção, para o caso das vulnera-bilidades RFI, LFI e RCE, e a utilização ou não dos modelos de teste estático e dinâmico.

Tabela 1. Funções e recursos dos scanners

\begin{tabular}{|c|c|c|c|c|c|}
\hline Scanner & RFI & LFI & RCE & Dinâmico & Estático \\
\hline Acunetix 6.5 free & & & & $\mathrm{X}$ & \\
\hline WebCruiser & & & & $\mathrm{X}$ & \\
\hline Shadow & & & & $\mathrm{X}$ & $\mathrm{X}$ \\
\hline Websecurify 0.7 & & & & $\mathrm{X}$ & \\
\hline Nikto v2.1.3 & & & & $\mathrm{X}$ & $\mathrm{X}$ \\
\hline PowerFuzzer & $\mathrm{X}$ & $\mathrm{X}$ & & $\mathrm{X}$ & \\
\hline Uniscan & $\mathrm{X}$ & $\mathrm{X}$ & $\mathrm{X}$ & $\mathrm{X}$ & $\mathrm{X}$ \\
\hline
\end{tabular}

Para a avaliação das ferramentas, sendo algumas delas proprietárias e de código fechado, foi preparado um cenário empírico, constituído de um servidor Web Apache e três arquivos PHP com as formas ma is simples de cada u ma das vulnerabilidades. No caso dos testes dinâmico e estático, a análise foi realizada através da atividade decada ferramenta no servidor Web, observando os registros doservidor Web Apache.

Como pode ser observado na Tabela 1, além do Uniscan, apenas o scanner PowerFuzzer detectou as vulnerabilidades RFI e LFI. No entanto, apenas o Uniscan foi capaz de 
detectar a presença das três vulnerabilidades alvo. Por fim, apenas três ferramentas fazem uso simultâneo das metodologias de testes estáticose dinâmicos, o Shadow, o Nikto e o Uniscan.

\subsection{CenÁ Rios Controlados}

Os cenários controlados possuem um número de vulnera-bilidades conhecidas, ou seja, os resultados possíveis e espe-rados dos scanners são previamente conhecidos. O cenário controlado 1contem três vulnerabilidades: RCE, RFI e LFI. O cenário controlado 2 é um sistema de blogque contém duas vulnerabilidades, uma $X S S$ e outraBlind SQL Injection. Os dois cenários foram preparados na plataforma Linux Ubuntu Server 10.04.3, com servidor Web Apache 2.2.15, PHP versão 5.3.3-1 ubuntu9.3 e Perl versão 5.10.1.

Tabela 2. Resultados dos testes nos cenários controlados $1 \mathrm{e} 2$

\begin{tabular}{|c|c|c|c|c|c|}
\hline Cenário Con trolado & \multicolumn{3}{|c|}{1} & \multicolumn{2}{|c|}{2} \\
\hline Scanner & RFI & LFI & RCE & XSS & Blind SQL-i \\
\hline Uniscan & 1 & 26 & 6 & 0 & 1 \\
\hline Acunetix & 1 & 2 & 0 & 3 & 6 \\
\hline Nikto & 0 & 0 & 0 & 0 & 0 \\
\hline WebCruiser & 0 & 0 & 0 & 0 & 0 \\
\hline Shadow & 0 & 2 & 0 & 0 & 0 \\
\hline Websecurify & 0 & 0 & 0 & 0 & 0 \\
\hline PowerFuzzer & 1 & 1 & 0 & 0 & 0 \\
\hline
\end{tabular}

Os números da Tabela 2 representam o número de combi-nações de testes detectados por cada ferramenta sobre as vulnerabilidades detectadas. Como exemplo,pode ser citada a vulnerabilidade LFI. O Uniscan realiza 43 testes distintos (detectou no sistema alvo apenas 26 formas de exploração) para esta vulnerabilidade, enquanto que o Acunetix realiza 5 (detectou duas formas de explorar o sistema alvo).

Como é possível observar, para os resultados do cenário controlado 1, o Uniscan foi o único scanner que cons eguiu detectar as três vulnerabilidades presentes. O Acunetix e o PowerFuzzer foram capazes de detectar as vulnerabilidade RFI e LFI.

Outro aspecto a observar é o fato de os scanners Shadow e PowerFuzzer tere m detectado as vulnerabilidades LFI e RFI, o que não ocorreu nos testes realizados para a Tabela 1. Is so comprova, ma is uma vez, a variabilidade nos resultados para uma mes ma ferramenta, executada em cenários diferentes, ou entre os diferentes scanners, conforme comprovado por algu-mas pesquisas [11, 12, 13].

O segundo cenário continha apenas duas vulnerabilidades, sendo uma XSS e outra Blind SQL-i. A ferramenta Acunetix obteve os melhores resultados, conseguindo explorar de dife-rentes formas as duas vulnerabilidades. O Uniscan detectou apenas a vulnerabilide Blind SQL-i devido ao fato de ainda não possuir um interpretador Javascript, o que reduz o escopo de pesquisa dentro do sistema alvo. Um dos trabalhos futuros previstos para a ferramenta é a inclusão de interpretadores para linguagens de scripting como Javascript e Actionscript.
A Tabela 3 apresenta os resultados dos cenários contro-lados 3, 4 e 5 . O cenário 3 representa um Plugin Annonces versão 1.2.0.0[29] para Workpress versão 3.3.2. Este plugin contém vulnerab ilidades LFI conhecidas[32,33]. O cenário 4 representa o sistema Supernews versão 2.6[30]. Este sistema Web contém uma vulnerabilidade de SQL Inject que pode ser explorada de diferentes formas[34]. O cenário 5 representa o sistema Membis versão 2.0.1[31]. Este sistema contém vulne-rabilidades Cross Site Scripting (XSS) e SQL Injection[35]. Todos os três sistemas dos cenários 3,4 e 5 foram instalados e avaliados sobre uma plataforma Ubuntu Server 11.04 com servidor Web Apache 2.2.17.

Tabela 3. Resultados dos testes nos cenários controlados 3,4 e 5

\begin{tabular}{|c|c|c|c|c|}
\hline Cenário Con trolado & 3 & 4 & \multicolumn{2}{|c|}{5} \\
\hline Scanner & LFI & SQL-i & XSS & Blind SQL-i \\
\hline Uniscan & 1 & 3 & 37 & 1 \\
\hline Acunet ix & 0 & 3 & 4 & 4 \\
\hline OpenVAS & 0 & 0 & 0 & 0 \\
\hline Arachni & 0 & 1 & 1 & 1 \\
\hline W3af & 0 & 0 & 0 & 0 \\
\hline
\end{tabular}

Nos cenários 3, 4 e 5, a lém do Uniscan e do Acunetix, foram testados três scanners de vulnerabilidades adicionais, sendo eles o OpenVAS [26], Arachni[27] e W 3af[28]. Como pode ser observado, mais uma vez, existem variações entre as ferramentas. Entretanto, considerando os testes empíricos e estatísticos realizados e apresentados neste artigo, as ferra-mentas Acunetix e Uniscan possuem um desempenho superior as demais na detecção e diagnóstico de vulnerabilidades emsistemas Web.

\subsection{CenÁ Rios Reais}

A utilização de cenários reais serve para comparar os resultados de todos os scanners sobre cenários do dia-a-dia, suportados por diferentes sistemas operacionais, como Windows Server, GNU/Linux e FreeBSD. A linguagem de programação também varia de um cenário para outro, incluindo PHP, ASP e Perl. Visando manter a privacidade dos sites testados, os nomes e endereços serão omitidos.

Como cenários reais, foram escolhidos aleatoriamente 29 sites, servindo de alvo para os scanners realizarem suas análises. Os sites estão distribuídos na Internet e localizados em diferentes países. Cadascanner foi executado para cada um dos 29 sites.Do conjunto de sitios analizados:(a) apenas $24.13 \%$ não apresentaram nenhuma vulnerabilidade; (b) 20.68\% apres entaramas vulnerabilidades LFI, RFI ou RCE; (c) $75.86 \%$ continham vulnerabilidades XSS; (d) $24.13 \%$ apres entaramproble mas de SQL-i; e (e) $41.37 \%$ continham a vulnerabilidade Blind SQL-i.

A seguir são apresentados dois exemplos, dentro dos 29 sitios avaliados, de sistemasalvo utilizados nos testes. As tabelas apresentam os resultados obtidos por cada um dos scanners.

O site A, cujos resultados são apresentados na Tabela 4, é um site sobre a cultura e o comércio chinês. O site utiliza a linguagem de programação Perl e é suportado por um 
servidor Web Apache 1.3.34. Como é possível observar, o Uniscan cons eguiu detectar ma is vulnerabilidades do que os demais scanners.Isto se deve ao fato do Uniscan ter mais e diferentes combinações de testes de vulnerabilidade do que as outras ferramentas. Além disso, apenas o Uniscan foi capaz de dete-ctar a vulnerabilidade RCE.

Tabela 4. Vulnerabilidades detectadas no site A

\begin{tabular}{|c|c|c|c|c|c|c|}
\hline Scanner & RFI & LFI & RCE & XSS & SQL-i & $\begin{array}{c}\text { Blind } \\
\text { SQL-i }\end{array}$ \\
\hline Uniscan & 0 & 72 & 151 & 906 & 0 & 23 \\
\hline Acunetix & 0 & 0 & 0 & 176 & 0 & 9 \\
\hline Nikto & 0 & 0 & 0 & 0 & 0 & 0 \\
\hline WebCruiser & 0 & 0 & 0 & 0 & 0 & 0 \\
\hline Shadow & 0 & 2 & 0 & 0 & 0 & 0 \\
\hline Websecurify & 0 & 0 & 0 & 20 & 0 & 0 \\
\hline PowerFuzzer & 0 & 0 & 0 & 0 & 0 & 0 \\
\hline
\end{tabular}

Tabela 5. Vulnerabilidades encontradas no site B

\begin{tabular}{|c|c|c|c|c|c|c|}
\hline Scanner & RFI & LFI & RCE & XSS & SQL-i & $\begin{array}{c}\text { Blind } \\
\text { SQL-i }\end{array}$ \\
\hline Uniscan & 0 & 0 & 0 & 5018 & 821 & 1532 \\
\hline Acunetix & 0 & 0 & 0 & 19 & 0 & 351 \\
\hline Nikto & 0 & 0 & 0 & 0 & 0 & 0 \\
\hline WebCruiser & 0 & 0 & 0 & 0 & 15 & 0 \\
\hline Shadow & 0 & 0 & 0 & 0 & 0 & 0 \\
\hline Websecurify & 0 & 0 & 0 & 3 & 22 & 0 \\
\hline PowerFuzzer & 0 & 0 & 0 & 0 & 0 & 0 \\
\hline
\end{tabular}

O cenário $\mathrm{B}$, cujos resultados são apresentados na Tabela 5,representa um site de uma academia de artes. O sistema utiliza a linguagem de programação ASP e é executando sobre um servidor Web Microsoft-IIS 6.0. Os scanners Acunetix, Uniscan e Websecurify foram os únicos a detectar a vulnerabilidade XSS. Os scanners Un iscan, WebCruiser e Websecurify detectaram a presença da vulnerabilidade SQL injection. Por fim, o Acunetix e o Uniscan detectaram a vul-nerabilidade Blind SQL injection. No somatório geral, indi-vidualmente, o Uniscan detectou o maior número de vulne-rabilidades e o maior número de combinações para exploração das vulnerabilidades.

A seguir, na Tabela 6, são apresentados os resultados totalizadospara cada ferramenta. Os números representam o total de combinações, de exploração da vulnerabilidade, dete-ctadas para o conjunto dos 29 sites. Pode-se notar que o Uniscan foi o único scanner que conseguiu detectar as seis vulnerabilidades, além de, na média, encontrar um maior número de combinações de exploração das vulnerabilidades existentes nos sistemasalvo.

Tabela 6. Resultados acumulados dos sites analisados

\begin{tabular}{|c|c|c|c|c|c|c|}
\hline Scanner & RFI & LFI & RCE & XSS & SQL-i & $\begin{array}{c}\text { Blind } \\
\text { SQL-i }\end{array}$ \\
\hline Uniscan & 1 & 79 & 257 & 8051 & 1055 & 2363 \\
\hline Acunetix & 1 & 0 & 0 & 923 & 9 & 389 \\
\hline Nikto & 0 & 0 & 0 & 0 & 0 & 0 \\
\hline WebCruiser & 0 & 0 & 0 & 20 & 60 & 4 \\
\hline Shadow & 0 & 2 & 0 & 1 & 0 & 0 \\
\hline Websecurify & 0 & 0 & 0 & 48 & 34 & 0 \\
\hline PowerFuzzer & 0 & 0 & 0 & 0 & 0 & 0 \\
\hline
\end{tabular}

É possível observar na Tabela 6 que apenas o Uniscan foi capaz de detectar a vulnerabilidade RCE. Outro ponto explicitado na tabela é o fato de dois scanners não terem detectado nenhuma vulnerabilidade nos sites analizados.

A partir dos resultados apresentados, pode-se afirmar que o scannerdesenvolvido contribui para a detecção e o diagnós-tico de vulnerabilidades em sistemas Web. Em alguns casos, a ferramenta desenvolvida apresentou resultados melhores que os scanners similares.

Um segundo, mas não menos importante, resultado posi-tivo do projeto foi o reconhecimento das potencialidades da ferramenta, levando a inclusão do Uniscan, desde a sua versão 3.2,na distribuição Linux BackTrack.Esta distribuição GNU/Linux é uma das que contempla a maior co mpilação de aplicativos voltados para a segurança e auditoria de sistemas. O número de utilizadores do Uniscan, no contexto do Linux BackTrack, é desconhecido. Entretando, sabe-se que o número de usuários da distribuição ultrapassa a marca de um milhão.

O Uniscan está disponível como ferramenta livre e gratuita[16]. As últimas estatísticas, de fevereiro de 2012, do próprio SourceForge, informam que o Uniscan já ultrapas sou a marca 6.000downloads, oriundos de mais de 100 pais es [17].

\section{Conclusão}

Scanners de vulnerabilidades são ferramentas importantes para a detecção e o diagnóstico de vulnerabilidades em sistemas Web. Entretanto, inexistem ferramentas capazes de detectar todos os tipos e combinações de exploração das vulnerabilidades existentes. Consequentemente, existeespaç o para novas pesquisas e desenvolvimentos. Neste sentido, o desenvolvimento do Uniscan procurou suprir algumas lacunas na detecção de vulnerabilidades em sistemas Web, com especial atenção para as vulnerabilidades LFI, RFI e RCE.

Os estudos realizados permitiram identificar características importantes em um scanner de vulnerabilidades, incorporadas ao Uniscan, como a utilização simultânea das metodologias dinâmica e estática. A prime irautiliza u m rastreador para navegar e coletar dados do sistema alvo de forma autônoma. Por outro lado, a segunda metodologiapermite identificar subsistemas e links do sistema alvo que não são rastreáveis por um crawler.

A arquitetura do Uniscané outro ponto forte e um dife-rencial em relaçãoa maioria das demais ferramentasanali-zadas. $\mathrm{O}$ fato de utilizar plug-ins fracamente acomplados torna o scanner mais flexível, dinâmico e extensível,eliminando a necessidade dealterações,na ferramenta, para a inclusão de novas funcionalidades ou novos testes de vulnerabilidade. Com uma arquitetura modular, o próprio usuário pode rapida-mente criar os seus próprios plug-ins. Esta característica possibilita ainda configurações dinâmicas, 
sob-demanda, onde o usuário pode escolher quais testes deseja executar sobre um determinado sistema alvo.

Combase nos experimentos e testes realizados, o Uniscan foi o único scanner a detectar todas as seis vulnerabilidades testadas, LFI, RFI, RCE, XSS, SQL-i e Blind SQL-i. Os números apresentados comprovam a sua capacidade de contri-buir na deteç̧ão e no diagnóstico de vulnerabilidades em sistemas Web. O fato de ser livre e aberto contribui para a sua utilização e para o desenvolvimento do conhecimento desta importante linha dentro da área de segurança da informação.

Finalmente, a ferramenta apresentada está em constante evolução através do desenvolvimento e da agregação de novas funcionalidades, plug-ins, recursos e características que possamserúteis para contribuir a inda ma is na detecção e no diagnóstico de vulnerabilidades em sistemas Web. As contri-buições da comunidade, at ravés do site do projeto[16], são importantes para a continuidade do desenvolvimento e da evo-lução do Uniscan.

\section{REFERÊNCIAS}

[1] Fong, E.; Okun, V. "Web Application Scanners: Definitions and Functions". 40th Annual Hawaii International Conference on System Sciences, Jan. 2007.

[2] Nguyen-Tuong, A.; Guarnieri, S.; Greene, D.; Shirley, J.; Evans, D. "Automatically Hardening Web Applications Using Precise Tainting". Security and Privacy in the Age of Ubiquitous Computing. In IFIP Advances in Information and Communication Technology, Springer Boston, 2005.

[3] Antunes, N.; Vieira, M. "Detecting SQL Injection Vulnerabilities in Web Services". Fourth Latin-American Symposium on Dependable Computing, pp.17-24, 1-4 Sept. 2009.

[4] Walden, J; Doyle, M;Welch, G. A; Whelan, M. Security of open source web applications. In Proceedings of the 3rd International Symposium on Empirical Software En gineering and Measurement. IEEE Computer Society, Washington, DC, USA, 545-553, 2009.

[5] Kals, S; Kirda, E; Kruegel, C.; Jovanovic, N. SecuBat: a web vulnerability scanner. In Proceedings of the 15 th international conference on World Wide Web. ACM, NY, USA, 247-256, 2006.

[6] Hui-zhong Shi; Bo Chen; Ling Yu; , "Analysis of Web Security Comprehensive Evaluation Tools". Second International Conference on Networks Security Wireless Communications and Trusted Computing, pp.285-289, 24-25 April 2010.

[7] Jensen, M.; Gruschka, N.; Herkenhöner, R. A survey of attacks on web services. Computer Science - Research and Development. Springer Berlin / Heidelberg, vol. 24, issue 4, 2009.

[8] Teodoro, N.; Serrao, C. "Web application security: Improving critical web-based applications quality through in-depth security analysis". International Conference on Information Society (i-Society), pp.457-462, 27-29 June 2011.
[9] Curphey, M.; Arawo, R. "Web application security assessment tools". IEEE Security \& Privacy, vol.4, no.4, pp.32-41, Jul-Aug. 2006.

[10] Arkin, B.; Stender, S.; Mcgraw, G. "Software Penetration Testing". IEEE Security \& Privacy, v. 3, n. 1, p. 84-87, 2005.

[11] Bau, J.; Bursztein, E.; Gupta, D.; Mitchell, J. "State of the Art: Automated Black-Box Web Application Vulnerability Testing". IEEE Symposium on Security and Privacy, pp.332-345, May 2010.

[12] Vieira, M.; Antunes, N.; Madeira, H. "Using web security scanners to detect vulnerabilities in web services". IEEE/IFIP International Conference on Dependable Systems \& Networks,pp.566-571, 2009.

[13] Le, H. T.; Loh, P. K. K. "Unified Approach to Vulnerability Analysis of Web Applications". The International e-Conference on Computer Science (IeCCS 2007), 14-23 Dec. 2007.

[14] Fonseca, J.; Vieira, M.; Madeira, H. "Testing and Comparing Web Vulnerability Scanning Tools for SQL Injection and XSS Attacks," 13th Pacific Rim International Symposium on Dependable Computing, pp.365-372, 17-19 Dec. 2007.

[15] Simmons, S.; Edwards, D.; Wilde, N.; Just, J.; Saty anaray ana, M. "Preventingunauthorized island ing: cyber-threat analy sis". IEEE/SMC International Conference on System of Systems Engineering, April 2006.

[16] Uniscan Project at SourceForge. http://sourceforge.net/proje cts/uniscan/. Último acesso em de fevereiro de 2012.

[17] SourceForge. Uniscan Project Download Statistics: All Files. URL:http://sourceforge.net/projects/uniscan/files/stats/timeli ne?dates $=2011-01-01+$ to $+2012-02-23$. Último acesso em fevereiro de 2012.

[18] Websecurify. Web Application Security Scanner and Manual Penetration Testing Tool. URL: http://www.websecurify.co m.Último acesso em fevereiro de 2012.

[19] Janus Security.WebCruiser - Web Vulnerability Scanner V2.5.0. URL: http://sec4app.com/.Último acesso em fevereiro de 2012 .

[20] SAFETY-LAB. Shadow Security Scanner. URL: http://www.safety-lab.com/.Último acesso em fevereiro de 2012.

[21] Kozlowski, M. Powerfuzzer. URL: http://www.powerfuzzer. com/. Último acesso em fevereiro de 2012.

[22] CIRT, Inc. Nikto2 | CIRT.net. URL: http://cirt.net/nikto2/. Último acesso em fevereiro de 2012.

[23] ACUNETIX. Website Security - Acunetix Web Security Scanner. URL: http://www.acunetix.com. Último acesso em fevereiro de 2012.

[24] ABYSSSEC. PHP Fuzzing In Action. URL: http://www.exploit-db.com/download pdf/12943. Último acesso em fevereiro de 2012.

[25] ZONE-H. Defacements Statistics 2010: Almost 1,5 million websites defaced, what's happening? URL: http://www.zone-h.org/news/id/4737/. Último acesso em fevereiro de 2012.

[26] OpenVAS. OpenVAP advanced Open Source vulnerability scanner and manager. URL: http ://www.openvas.org/. Último 
acesso em junho de 2012.

[27] Arachni. Arachni - web application security scanner framework. URL: http://arachni-scanner.com/. Último acesso em junho de 2012.

[28] W3af. W3af Web Application Attack and Audit Framework.URL: http://w3af.sourceforge.net/. Último acesso em junho de 2012.

[29] WordPress.org. Wordpress Plugin Annonces 1.2.0.0. URL: http://wordpress.org/extend/plugins/annonces/. Último acesso em junho de 2012.

[30] Pontes, F. da S. Supernews (sistema de Notícias baseado em PHP e Mysql). URL: http://phpbrasil.com/script/vT0FaOCy SSH/supernews. Último acesso em junho de 2012.

[31] Membris. Membris 2. URL: http://www.membris.fr/. Último acesso em junho de 2012.

[32] Offensive Security. Wordpress Annonces Plugin 1.2.0.0 Remote File Inclusion. URL: http://www.exploit-db.com/ex ploits/17863/. Último acesso em junho de 2012.

[33] IBM Internet Security Systems (Ahead of the threat). Annonces Plugin for WordPress uploadPhoto.php remote file include. URL: http://xforce. iss.net/xforce/xfdb/69932. Último acesso em junho de 2012.

[34] Offensive Security. Supernews $<=2.6 .1$ (noticias.php cat) SQL Injection. URL: http://www.exploit-db.com/exploits/18 961/. Último acesso em junho de 2012.

[35] Offensive Security. Membris v2.0.1 Multiple Vulnerabilities. URL: http://www.exploit-db.com/exploits/18970/. Último acesso em junho de 